\title{
Normal Human Cells Acquiring Proliferative Advantage to Hyperplasia-Like Growth-Morphology: Aberrant Progeny Cells Associated with Endopolyploid and Haploid Divisions
}

\author{
Kirsten H. Walen ${ }^{1}$ \\ ${ }^{1}$ CROMOS, Richmond, CA, USA \\ Correspondence: Kirsten H. Walen, CROMOS, 763 Ocean Avenue, Richmond, CA 94801, USA. Tel: \\ 001-510-234-3375. E-mail: kwalencromos@gmail.com
}

Received: July 3, 2013 Accepted: July 22, 2013 Online Published: July 29, 2013

doi:10.5539/cco.v2n2p19 URL: http://dx.doi.org/10.5539/cco.v2n2p19

\begin{abstract}
Today, cancer-patients can seek individual genomic tailored therapy, but at escalating costs. However, cancer-incidence reduction would reduce such health-cost. The essence of this report is a cell-culture experimental approach for the production of genetically unstable cells from normal cells which with accumulation of "mutations" might reveal early, neoplastic-like pathology. Summarily the experimental focus was on: 1) establishment of a simple nutritional method (amino acid deprivation) for young-cell-response with depoly-ploidization of endopolyploid cells to diploid CIN-cells, 2) a first time observation of a fast growing new, small cell-type, appearing time-correlated with haploid segregations in normal, fibroblast metaphases and 3) growth characteristics of the progeny cells in 1) and 2) which from their young-cell derivation, could be growth-extended in cultural passages. This would hypothetically model early tumorigenic progression from normal cells. These studies revealed gain of proliferative advantage (GPA), multilayered, changed patterns of growth, focal 3-D cell-heaps with cell polarity change, and star-like mitotic forms. Comparatively, these developments were typical of hyperplasias. The transiency of the originating genome reductive mechanisms may have prevented earlier detections. It is discussed that present growth-changes were responses to homozygous-caused loss of heterozygosity $(\mathrm{LOH})$ of putative pro-proliferation genetics, whereas leukemic and some solid tumor cells showed hemizygous-caused LOH in near-haploid clonal proliferations. The practical outcome is an experimentally simple, nutrition-based model system for SNP-based haplo-karyotyping of the two aberrant cell-types with GPA, which is a cancer, fundamental trait. Never before have cellular mechanism been shown to initiate in normal, human cells, neoplastic-like proliferative responses.
\end{abstract}

Keywords: primary young cells, aneuploidy, chromosomal instability (CIN), genome reductive division, endopolyploid inherited division traits, amino acid deprivation, three dimensional growth, nuclear rounded shape-change, haploid chromosome groupings , karyology

\section{Introduction}

Mitotic karyology is presently a complementing method to karyology by single-nucleotide-polymorphism (SNP) array studies in tumorigenesis (Yang et al., 2011, Fan et al., 2011; Peters et al., 2012). SNP has the advantage of interphase cell-use and of resolving "cryptic" genomic changes such as single nucleotide change, small deletions and duplications, and also whole chromosomal and segmental uni-parental disomy (UPD) with homozygous-caused loss of heterozygosity (LOH) (Fitzgibbon et al., 2005; Gondek et al., 2008). Does this mean that cytogenetics in the near future will be even more passé than it has already become? - "At times the focus on molecular genetics is so strong that all other means of gaining relevant information seem to have been forgotten; surely, the fascination with new things and toys is one of the most profound character trait of man" (Heim and Mitleman, 1995).

Gross cellular data however, can unveil the etiology in normal cells for diseases without such knowledge as for example, in cancer development (Vogelstein \& Kinzler, 1993). This assumption rests on informational gathering by cytogenetics/cytology/-morphology-methods, similar in approach to cancer pathological diagnosis. Only thereafter, can a molecular "diagnosis" be obtained by use of biopsies or in vitro model systems containing the cellular structural changes. These latter data can disclose therapy-relevant pathways and also 
vaccine-possibilities for reduction in cancer incidence. For "origin" of cancer-disease, ideas of molecular events have been constructed for mouse model-systems (Coschi \& Dick, 2012), but no similar normal cell culture-derived system have been clearly delineated (disregarding immortalization - see Blagosklonny, 2003). On the gross cellular level it was once believed that mitotic unequal distribution of chromosomes to daughter cells was the initiating event (Boveri, 1914). This idea is also currently fostered through aneuploidy, which assumes that such events (Goodyear et al., 2009) originate from the normal mitotic phenotype (Manchado et al., 2012). Pathologist Von Hansemanns' cancer observations were mostly on cell-division phenomena which are rarely observed in routine pathology, from which he emphasized different "forms" of mitosis, and the morphological variety of asymmetric chromosomal distributions (Bignold et al., 2007). In contrast to Boveri, he therefore, expected cancer initiating events to show a different "type" of mitosis (Hardy and Zacharias, 2005). These distribution error-events are today linked to gain of proliferative advantage (GPA) from aneuploidy and chromosomal instability (CIN) (Lengauer et al., 1998; Geigl et al., 2008; Backhoum \& Compton, 2012; Coschi \& Dick, 2012). But, aneuploidy in normal cell populations shows reduced proliferative ability, and those are the cells for the origin of cancer (Sheltzer \& Amon, 2011). The question is therefore, whether there are known abnormal cellular events that can originate in normal cells and, lead to aneuploidy and CIN-cells? The answer is yes, the mechanism of endopolyploid genome reductive divisions (Wolfe, 2001) produce genome unstable, diploid progeny cells (Walen, 2006, 2007a,b; 2010; 2012; Lee et al., 2009; Erenpreisa et al., 2011).

Although polyploidy is mentioned in a cancer-connection in many reports, there is no distinction between ordinary polyploidy (e.g., $4 \mathrm{n}$ euploidy from 4 haploid genomes) and endopolyploidy with 4-chromatid chromosomes, which segregate meiotic-like with genetic consequences for proliferating progeny cells. Endotetraploid cells originate by G2 chromosomes (2 chromatids and 2 DNA helixes) skipping mitosis to a second S-period, which produce so-called diplochromosomes, recently confirmed by Davoli et al., (2010). Contrary to mitosis, this division-system is perpendicularly oriented relative to the cytoskeleton axis, which was reported for oral cancers (Saunders et al., 2000). This non-mitotic division splits maternal and paternal diplochromosomes into bichromatid chromosomes by co-segregating whole complements. The resultant $\mathrm{G} 2 / 2 \mathrm{n} / 4 \mathrm{C}$, genomes mostly formed resting cells, which is a genetic constitution ["4N (G2 tetraploid)"] in Barretts' esophagus, a pre-cancerous conditions (Galipeau et al., 1996; Barrett et al., 2003). Activation of these latter cells to mitotic activity with change to esophagus cancer was recently demonstrated by shared molecular data (Agrawal et al., 2012). It is however, unknown whether these precancerous cells can immediately continue with a second division into propagating, unstable paradiploid cells as occurred with moderate frequency for in vitro cells (Walen, 2007a,b; 2009; 2012).

The attainment of CIN for these latter cells originated from inheritance of endopolyploid special division traits (Walen, 2012). For some primitive unicellular organisms, the endo-derived reductive division-system is a mitotic-meiosis, and is also a perpendicular, complement co-segregation system (Raikov, 1982; Hunding, 1981; see Discussion). Naturally, for these organisms the goal is to pass-on to the progeny, unfailing division-traits for their reproduction. And interestingly, the endo-derived genome reduced human, progeny cells demonstrated several abnormal division-behaviors, linked to endo-division traits, as for instance, co-segregation of whole genomes in prophase cells. Another trait was mild chromosomal loss/gain from dysfunctional centromeres (Bakhoum et al., 2009) (co-segregation-associated defects in centromere bending-capacity affecting proper spindle attachment, Walen, 2009) leading to miss-behavior in anaphase (e.g., bridges/non-disjunction). Similar genomic co-segregation was reported for endopolyploid trophoblastic cells (Zybina \& Zybina, 1996; Zybina et al., 2005), which suggest evolutionary conservation of this primitive "meiotic-like" division system in mammalian cells, leading to variable CIN-consequences of progeny cells (i.e., endo-derived transmuted diploid cells - E-dTMCs). Inheritance-caused improper mitotic/meiotic divisions are known from both plants and animals (Swanson, 1957), and are a new concept for mitotic generational, production of genetic variability in normal cell populations. This theory has not been tested for continuously propagating E-dTMCs, which is an objective in the present study. Hypothetically, accumulated mutations might be expressed in growth-changes similar to assumed "progression" changes, predicted by the mutation theory (Lengauer et al., 1998).

Up-to-now proliferation of E-dTMCs had been limited to a few generations in pre-senescence, where endopolyploidization occurred associated with eroded telomeres (i.e., genomic damage) (Walen, 2006, 2007a,b; Davoli et al., 2010). An experimental method for induction of endopolyploidization in young human cells with long telomeres became the main objective for the present study. This method should preferentially avoid specific "mutable" agents (carcinogens, X-ray), and have relevance to every-day living, which brought up the saying, "you are what you eat". Epidemiology of many types of cancers (Lipkin, 1988) has certainly shown nutrition as a factor, and recently obesity was claimed to have increased risk of cancer (Tee \& Blenis, 2005). 
Nutrition-associated genomic changes were reported for in vitro serum starved human quiescent cells, which produced tumors in mice (Zhang et al., 2006). These notions plus some earlier results from amino acid deprivation (AAD) (Freed \& Schatz, 1969; Phillips et al., 2003) led to exposures of young, primary, human diploid cells (L645 and WI-38) to growth-medium deficient in glutamine (Deberardinis \& Cheng, 2010). The earlier studies from multiple different AA-deficiencies had shown endopolyploidization, giant cells, chromosomal aberrations and breakage. The authors suggested that AAD-exposures resulted in stalled replication-forks from lack/insufficiency of DNA-associated replication proteins.

In the present young-cell study endopolyploid divisions occurred in recovery growth (see $M \& M$ ) with genome reduction to interphase $2 \mathrm{n} / 4 \mathrm{C}$, cells and to proliferating diploid E-dTMCs. But about one day prior (in recovery-growths) to these occurrences a haploid grouping process were observed in normal metaphase cells, showing separations of 23 and 23 (or near 23) chromosomes from each other. The haploidization process was in the first waves of recuperated mitotic fibroblast cells, which was time concurrent with the appearance of a new, small cell-type (cause and effect?) with uniformly smaller roundish-shaped nuclei compared to the larger fibroblastic oval shape. These new cells also showed proliferation and were called, fibroblast derived prolific cells (F-dPCs), and were included with E-TMCs in expanded growth characterizations. The results showed hyperplastic-like growth from both cell-types (i.e., gain of a proliferative advantage - GPA) with changed growth patterns associated with focal three dimensional (3-D) growths. These results are discussed with comparisons to similar growth-characteristics in hyperplasias, and to the remarkable ability of leukemic cells to proliferate with a near-haploid complement.

\section{Materials and Methods}

For the two primary lung diploid, cell strains, L645 and WI-38, used in this study, all essential routine maintenance methods for extended passage (p) growth have been described several times (Walen, 2010; 2011, 2012). This study maintained the routine with Eagle's minimum essential medium supplemented by $10 \%$ fetal bovine serum (fbs), 100 units $/ \mathrm{ml}$ penicillin/streptomycin and $3 \mathrm{mmol}$ glutamine, and passages were made from a 1 to 4-dilution. The experimental design for induction of endopolyploidization in young passage-growth (L645p17/18, WI-38p19/20) was as follows: one day flask-growth (from 7-800,000 cells in T25 flasks) after 1 wash with Hank' balance salt solution was exposed to growth medium without glutamine and fbs reduced to $2 \%$ (M-) for 2, 3, 4 days. Such cultures were allowed 1 day recovery in complete medium $(\mathrm{M}+)$ before they were suspended by trypsin/versene solution for chamber-slide seeding (3-4000 cells). Several slides were exposed 5 minutes to hypotonic solution of $0.4 \%$ sodium citrate and $0.4 \%$ potassium chloride for karyology of grouped chromosomes. All slide cultures for were harvested by fixation (3:1 - Carnoy's solution) after 1,2, and 3 days (total recovery growth 2, 3, 4 days) in $\mathrm{M}+$. Flask cultures from 2 and 4 day M- exposures were passaged weekly with 1: 3 or 1: 4 dilutions which was reduced to one each from the two cell strains in late passages approaching senescence. Chamber slide cultures were made from different passage-growths. Gain of proliferative advantage for the two aberrant cell-types from their $3^{\text {rd }}$ passages were tested by seeding of flask cultures with 7-800,000 cells compared to similar flasks with control fibroblast cells. Two flasks from each cell-type were counted on day 4 of growth and another each on day 7. Additionally, from 3-day M- and 3-day M+ from which both "new" cell-types and fibroblasts co-proliferate in extended passage growths, the presence of fibroblast growth at the $3^{\text {rd }}$ passage was evaluated on chamber-slides. In all, 6 different M- exposures were sampled and examined in 6 sets of duplicate chamber slides. These slides were stained by $2 \%$ Giemsa in $\mathrm{pH} 6.8$ sodium and potassium phosphate buffer solution, followed by Coverbond cover-slip application. These preparations were examined in a Zeiss compound microscope with an attached Zeiss camera for $36 \mathrm{~mm}$ film. In all, about 150 photographs were taken from x16, x25, x40 and x100 objectives and enlarged respectively: 396x, 619x, 990x and 2475x. The karyotypes were from metaphase enlargements onto $8 \times 12$ photographic sheets.

\section{Results}

Several different experiments with both L645 and WI-38 primary, fibroblast, female, lung cell-strains were exposed to 2, 3 and 4 days to amino acid deficiency (AAD: glutamine) and changed to complete recovery-medium with various times of harvests ( 2 to 4 days) (see M\&M). Strain differences had been experienced earlier with WI-38 cells having more robust growth capacity (population doublings), and with more "violent" senescence-associated death than for "milder" L645 cells. The present study used WI-38 cells to verify $\mathrm{AAD}$ responses of L645-cells, and most of the illustrative material was selected from comparing photographs, which often, L645- cells showed with greater clarity. Both cell strains exposed to AAD showed in recovery-harvests endotetraploid reductive division to earlier described E-dTMCs and a new small, cell-type never before seen in L645 and WI-38 cell cultures. The timing of the small cells' appearances was concurrent with a haploid grouping process in diploid metaphases, also never observed before. 
In three random tallies of 50 rosette metaphases ( 2 for L645 and 1 for WI-38 cells) on in situ harvested chamber slides the number of haploid symmetric groupings to $23-23$ in metaphase cells were $7,5 \& 5$ and 5 groupings being obvious asymmetric. This becomes a grouping mean of $14.7 \%$, which agrees with the plant-assessment of about 12\% (Huskins, 1948). The actual dis-tribution of chromosomes during the grouping process was counted on photographs from selected cells with the least chromosomal overlaps, sometimes only possible for one group. In such cases this number was subtracted from 46 which would give the number of chromosomes in the second group. Forty such cells, 20 from each cell strain, show a very narrow distribution around 23-23 (Table 1, there were no strain differences and the counts were totaled). Several chamber slides were treated with "mild" chromosomal spreading solution for in situ karyology of the grouped chromosomes, which either had no effect or resulted in intermingling of the two groups.
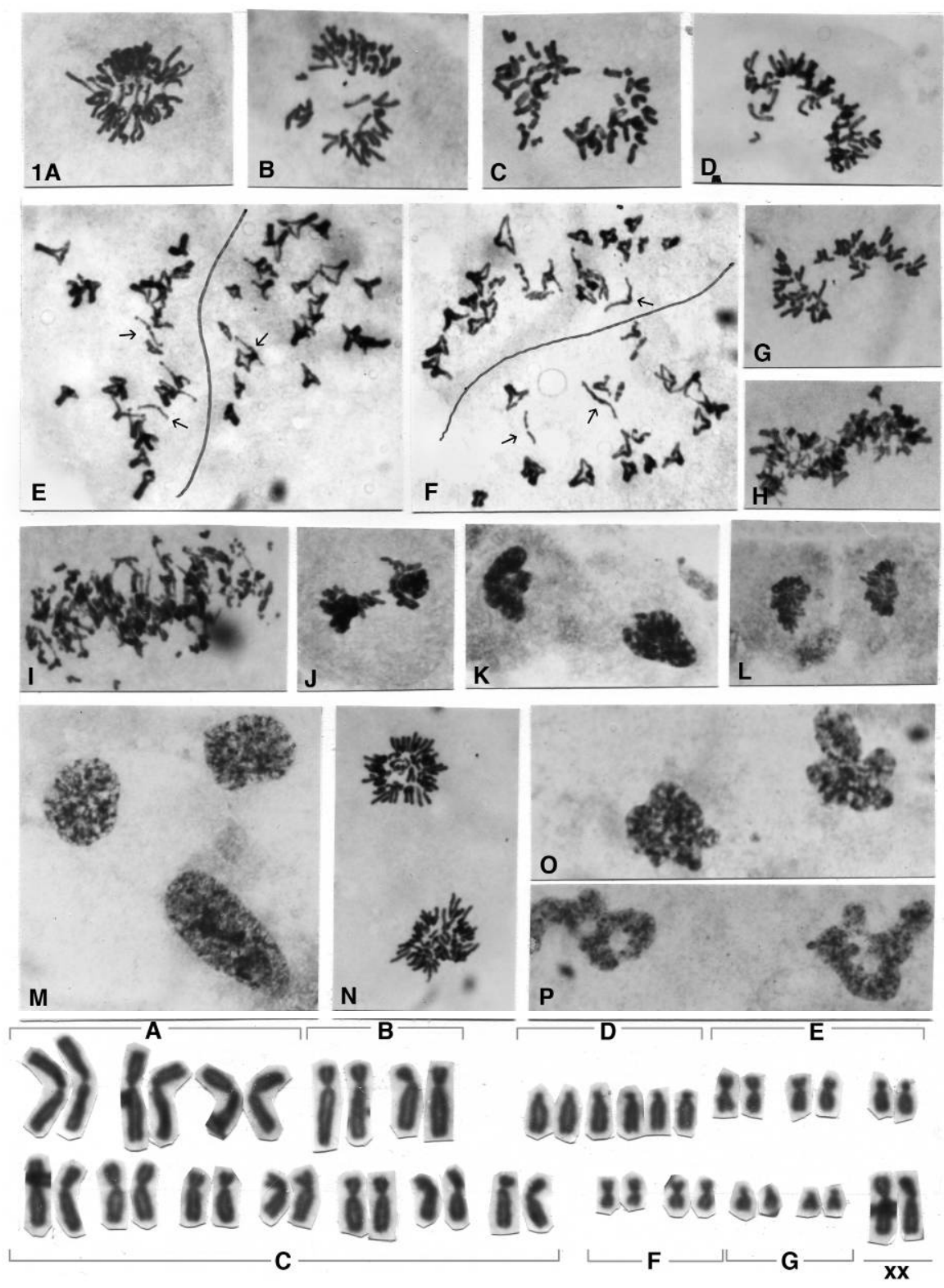

Q

Figure 1. Diploid metaphase cells with haploid groups. (A) a metaphase rosette figure. (B-D,GH) haploid groups with retained "half-moon" rosette morphology. (L) rosette-morphology and cytokinesis. (EF) separating chromosomal groups with sister chromatid exchanges into single thin, chromatid strands, connected in the centers by telomere-associations (arrows). (I) cell, side-view of a metaphase showing chromosome breakage and sister chromatid exchanges. (JK,M,OP) dysmorphic telophase groups compared to round nuclear shapes (M), $1 / 2$ size smaller than the normal oval, fibroblast nucleus. (N,Q) small, star-like telophase (compare to size of E-dTMC in $4 \mathrm{AB}$ ), and a karyotype from $3^{\text {rd }}$ passage F-dPC growth. All illustrations enlarged $2475 \mathrm{x}$ 
Figure 1 shows highlights from the haploid grouping process from representative illustrations. A rosette figure (Fig. 1A), possibly in beginning phase of halving into two groups of chromosomes, as seen in 1B, and $1 \mathrm{C}$ with disarrayed half, rosette forms. Size-wise these latter two groupings indicate symmetric groupings $(+/-1,2$ chromosomes). Retained half-sized rosette configurations (Figs. 1D,G,H,L) were a repeated observation with the average rate of one in every 3 groupings. Of note is that these haploid groups proceeded with cellular events for cytokinesis (Fig.1L). Some recovery periods (2-3 days) indicated DNA damage by sister strand crossover repair-figures (Figs. 1E,F), and in side-views, metaphases showed breakage (Fig. 1I). Interestingly, the grouping activity proceeded while the crossovers resolved into single, thin chromatin threads, showing telomere associations in the centers (Fig.1E,F, arrows).Typical of the grouping-progression was chromosome condensation to very uneven shaped interphase nuclei (Figs. $1 \mathrm{~J}, \mathrm{~K}$ ), but the formation of roundish nuclei $1 / 2$ sizes smaller than fibroblast nuclei were also present (Fig. 1M). From these grouping events, especially asymmetric, it was assumed that there would be noticeable presence of dying cells, but such was not the case. Several slide-screenings from different AAD-time exposures and different recovery-periods yielded not much more than presented in figures 1O,P. This extraordinary result either pointed to high survival-rate or a low rate of asymmetric chromosomal distribution. The latter was supported by the absence of apoptosis fragmented nuclei and of micronuclei, and also by the narrow range of asymmetric groupings (Table 1).

Table 1. Number chromosomes in haploid groups from 20 L645 and 20 WI-38 normal diploid cells

\begin{tabular}{lllllllllllllllllllllll}
\hline & 13 & 14 & 15 & 16 & 17 & 18 & 19 & 20 & 21 & 22 & 23 & 23 & 24 & 25 & 26 & 27 & 28 & 29 & 30 & 31 & 32 & 33 \\
\# cells & 1 & 0 & 3 & 1 & 3 & 4 & 3 & 3 & 5 & 7 & 10 & 10 & 7 & 5 & 3 & 3 & 4 & 3 & 1 & 3 & 0 & 1 \\
\hline
\end{tabular}

Example: the smallest group contained 13 chromosomes which on the assumption of a total of 46 chromosomes (normal cells) result in 33 chromosomes for the larger uncountable group.

In these harvest periods ( 2 and 3 days in $\mathrm{M}+$ ) small mitotic cells with changed form to star-like divisions from metaphase to telophase were present (Fig. 1N compare fig. 2D - normal telophase). Their sudden appearance in all $6 \times 2$ slide harvests was time concurrent with the short time-window in which the haploid groupings occurred (cause and effect?). These events were almost entirely restricted to the first wave of recuperated mitotic activity, which consisted mostly of normal fibroblast mitoses, dividing normally, back to polarized fibroblasts with oval shaped nuclei. The new, small cell-type with rounded nuclei first appeared as pairs of rounded cells among mitotic fibroblasts, scattered aroung the cultural surface, but seemingly not exceeding the grouping rate $(\sim 14 \%)$. Only about 3 to 4 areas of proliferating small cells were observed, suggesting poor viability or selection of genotypes from the pairs of rounded cells. These proliferating cells with star-like form of mitosis persisted in flask cultures split 1 to 3 for the next passage, which after 7 days growth contained about half and half mixtures of small and fibroblast cells. In the following passage the small cells were the major cell population with near-to-absence of fibroblastic cells, they had outgrown the cell of origin. Six different recovery periods showed this type of growth behavior for the new small cell-type which had gained proliferative advantage at "birth", and were called fibroblast-derived prolific cells (F-dPCs). In 3-day proliferative assessments of $3^{\text {rd }}$ passage cells (see M\&M) compared to similarly seeded control, fibroblast cultures, hemocytometer counts of F-dPCs for two cultures were 3.4 and $3.7 \times 10^{6}$ whereas controls showed 1.7 and $2.2 \times 10^{6}$ cells. In 7-day cultures, the F-dPCs "packed" the cultures with seemingly multilayered growth, from close to 5 million more cells than in control flasks (normal: 4-5 $\times 10^{6}$ ). Furthermore, chamber-slides for in situ evaluation of the presence of proliferating fibroblasts in the $3^{\text {rd }}$ passage from originally co-proliferating E-dTMCs, F-dPCs and fibroblasts cultures did not reveal normal fibroblast mitotic figures (Fig. 4D).

The F-dPCs cell sheets in passages consisted of homogeneous roundish-shaped cells that could grow over each other (Fig. 2B,E), and in some cases seemed to start growth from a butted end growing with increase in rows of cells in a "streaming" growth pattern (Fig. 2C,E,F). A material substance appeared to cover the sheets giving poor staining resolution to the rows of cells (Fig. 2F), but phase contrast photography resolved this issue to some degree (Fig. 2E). Of note is that these cells can form polyploid cells (Fig. 2D, arrow). Undeniably, these F-dPCs were distinctly different in morphology, nuclear shape, form of mitosis and growth pattern from the fibroblastic, more heterogeneous cell populations (Fig. 2A). Most importantly, these new cells had acquired hyperplasia-associated traits: - multilayered growth and GPA over cells of origin. A chromosome number assessment of metaphases in figure 2C-growth showed 46 chromosomes in 5 cells (naturally spread metaphases), and a karyotype was prepared from one such cell, sorted into A, B, C, D, E, F, G and XX groups (Fig. 1Q). There were no gross chromosomal changes. (Illustration is out-of-order, because of no space in figure 2.) 

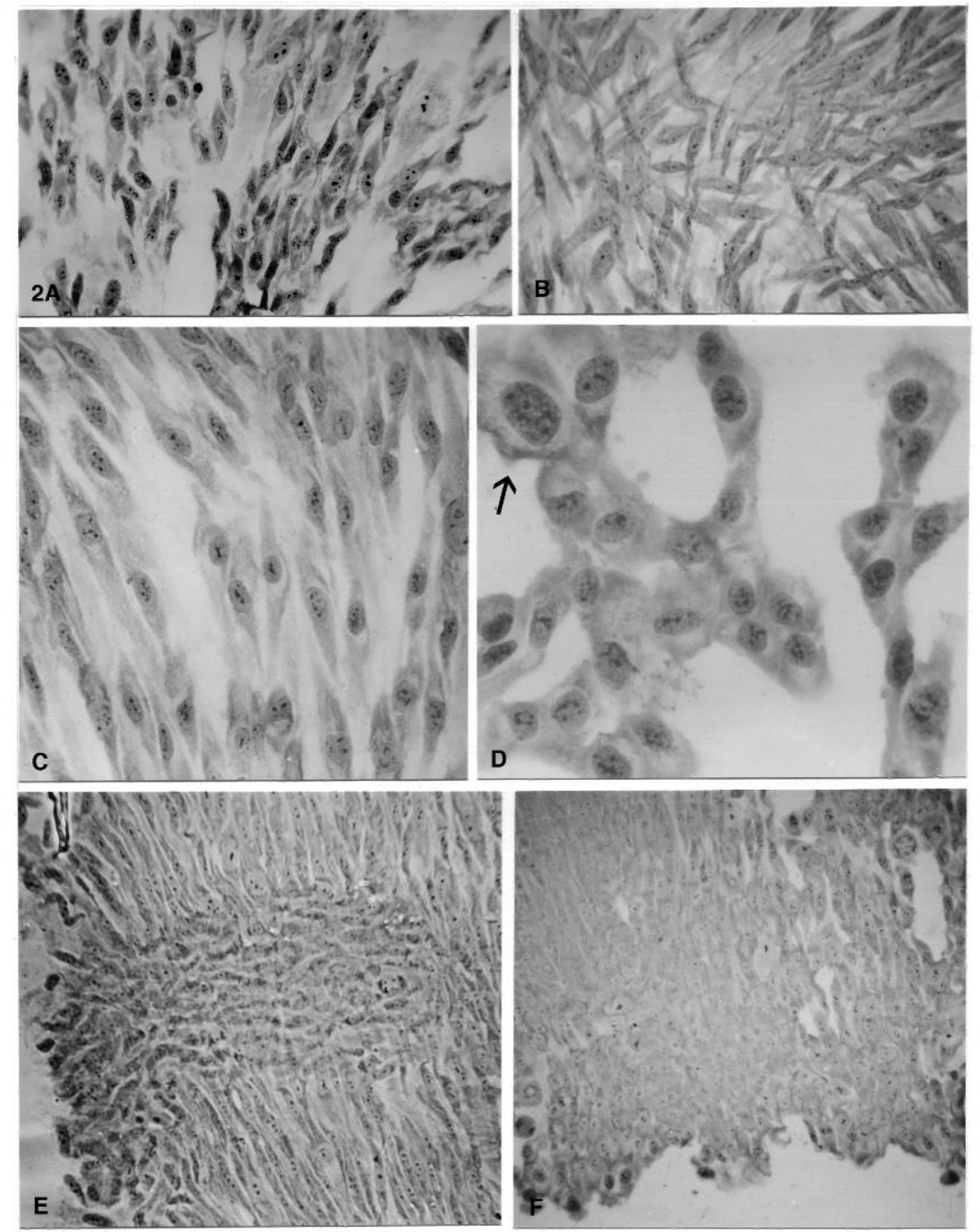

Figure 2. Proliferative behavior and morphology of F-dPCs in extended passage growth. (A) normal -control fibroblastic polarized growth from start-passage of AAD treatment. (BE) multilayered growth. (CD) change to homogeneity of nuclear-size and cell-shape, one cell with polyploidy (arrow). (EF) streaming growth pattern of uniform, spindle shaped cells, and presence of a substance (F) preventing normal stain uptake (see text). Enlargements: (AB,EF) 396x; (C) 619x; (D) 990x

Endotetraploid cells with reductional division to E-dTMCs appeared with low incidence in 3 day and abundantly, in 4 days recovery-growths. These cells, also with rounded nuclei (Fig. 3A) started proliferation more or less in step with the normal fibroblasts, and showed earlier, described (Walen, 2011, 2013) cytoskeleton-associated skewed, star-like mitotic-sequence from metaphase to telophase (Fig.3C, arrow). The E-dTMCs persisted in diluted (1:3) passages and by passage 3 and onward became the predominant cell population as compared to presence of original fibroblastic proliferation. This incremental gained growth increase at the $3^{\text {rd }}$ passage compared to control fibroblast was tested from similarly seeded cultures on day 4 and 7 of growth. Two E-dTMC cultures showed only 600,000 to 800,000 more cells than the controls. The 7-day culture was not "packed" with cells as in F-dPCs-flasks, and showed only about 1 and 1/2 million more cells as compared to control cells. The chamber slide cultures from the $3^{\text {rd }}$ passage did not show fibroblast-mitosis. Interestingly, this passage and onward also showed "streaming" growths of arrays of cells, but with more size and shape heterogeneity as compare to the F-dPCs (Fig. 3B, arrows). Exceptional for E-dTMC-streams were their relatively rarer $\left(\sim 3-5\right.$ heaps in $10^{-6}$ cells) associations with 3-D heaped cell-growth, showing disoriented cells from cell polarity change (Fig. 3D,E). These heaps were also covered by a substance, seemingly denser than that for F-dPC-streams (Fig.3E,F), but with clearly visible star-like mitoses at the edge of growth and also inside the growths (Fig. 3F, arrows). (Normal fibroblast growth is highly polarized and always in a single, layered 
cell-sheet with a passage-constant $2-3 \%$ of all mitoses being regular polyploid, which indicate no proliferative advantage of this type of polyploidy over diploid cells - Hayflick and Moorhead, 1961)

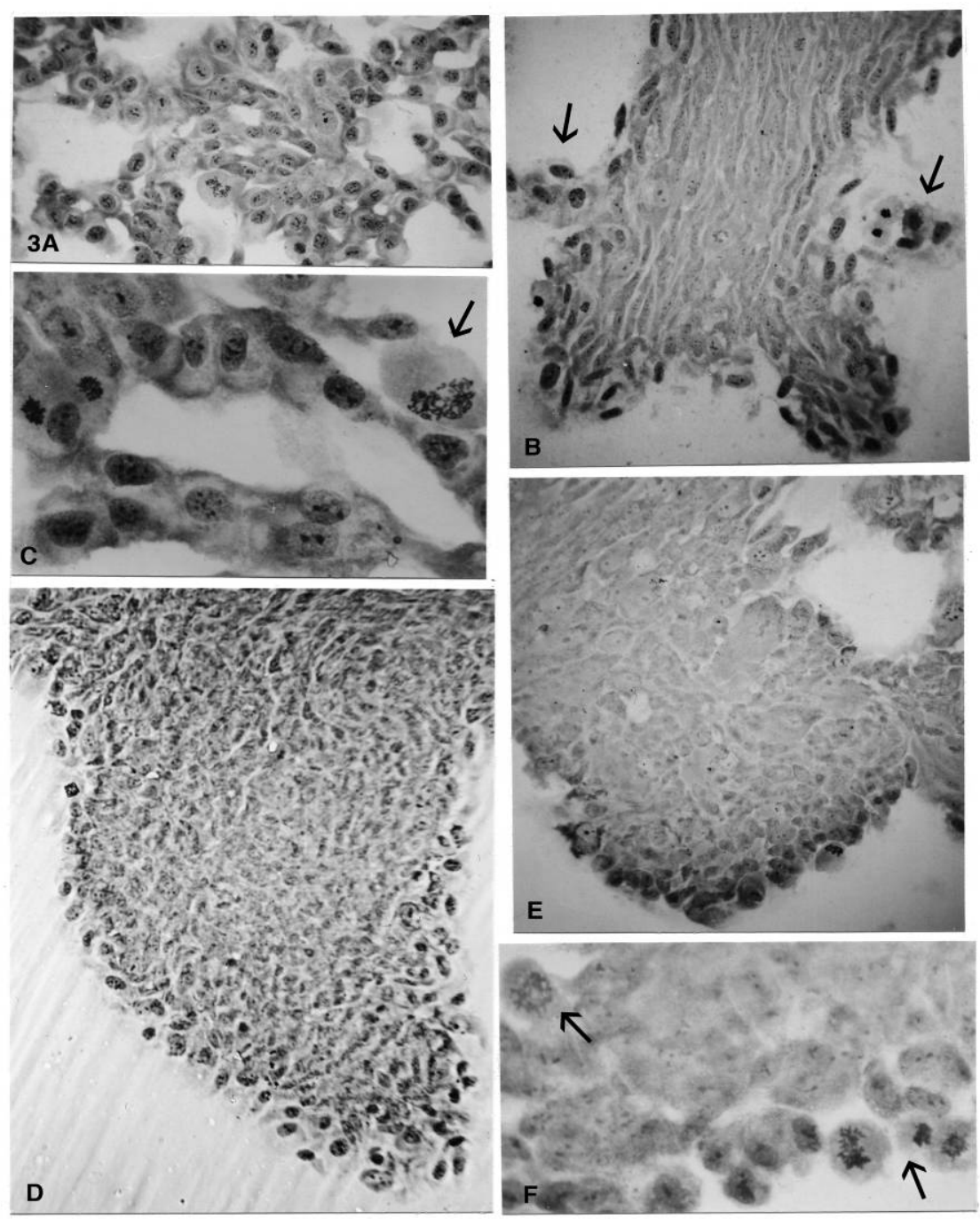

Figure 3. Proliferative behavior and morphology of E-dTMCs in extended passage growth. (A) induced, beginning clonal proliferated, large, rounded cells. (BC) nuclear-size heterogeneity (B, arrows) and star-like skewed division relative to the cytoskeleton axis (C, arrow), and "streaming" growth-pattern (B). (DEF) focal, three dimensional growths with cell polarity change (D), star-like mitosis (F arrows), and presence of a substance preventing normal stain uptake (EF) (see text). Enlargements: (AB,DE) 396x; (C,F) 990x

Figure 4 compares star-like divisions from E-dTMCs in beginning growth (recovery day-4), to stars from extended passage-growth (3-4 passages). Figures 4A,B are typical stars at beginning ana- to telophase. Figure 4C demonstrate that this segregation pattern is not always perfect, estimated to occur in 1-2\% of the "normal" star-divisions. But, the overall importance is the striking difference between telophase stars and normal mitotic telophases (Figs. 4B,C compare 4D). The perpendicular orientation of endopolyploid division relative to the cytoskeleton axis is shown as inserts in figures 4F,G (see Discussion). In passage growth the streams showed diploid and tetraploid rosette figures with premature chromatid separations (Fig. 4E,F,I), and a different form of early anaphase separated genomes (Fig. 4G,J) as compared to figure 4A. Disarrayed or early aberrant segregations (Fig. $4 \mathrm{H}$ ) were also present. Additionally, the co-segregation process in endo-reductive divisions was expressed for what appears to be a tetraploid prophase nucleus (Fig. 4K). These examples from one streaming E-dTMC growth indicate polyploidy and cell-division-associated possibilities for chromosomal distribution-mistakes. Furthermore, change in nuclear shape to irregular circumference (achantosis in pathology) 
(Figs. 4F,G) is a new feature associated with expanded E-dTMC- proliferation. Karyology and chromosome counts were only possible in "looser" growths of E-dTMC-streams where naturally spread metaphases were observed. Seven cells showed 46 chromosomes and one, with only one overlap was arranged in pairs according to A,B,C,D,E,F,G and sex chromosomal groups (Fig. 4L). No obvious abnormalities were evident. Neither cell-type (F-dPCs and E-dTMCs) indicated an immortal status, because lysosomal activity was present in late extended passages (29-33), which suggest senescence-occurrence. But these happenings need further studies, together with inquiries into the growth-destiny and karyotypes of cells from 3-D growths
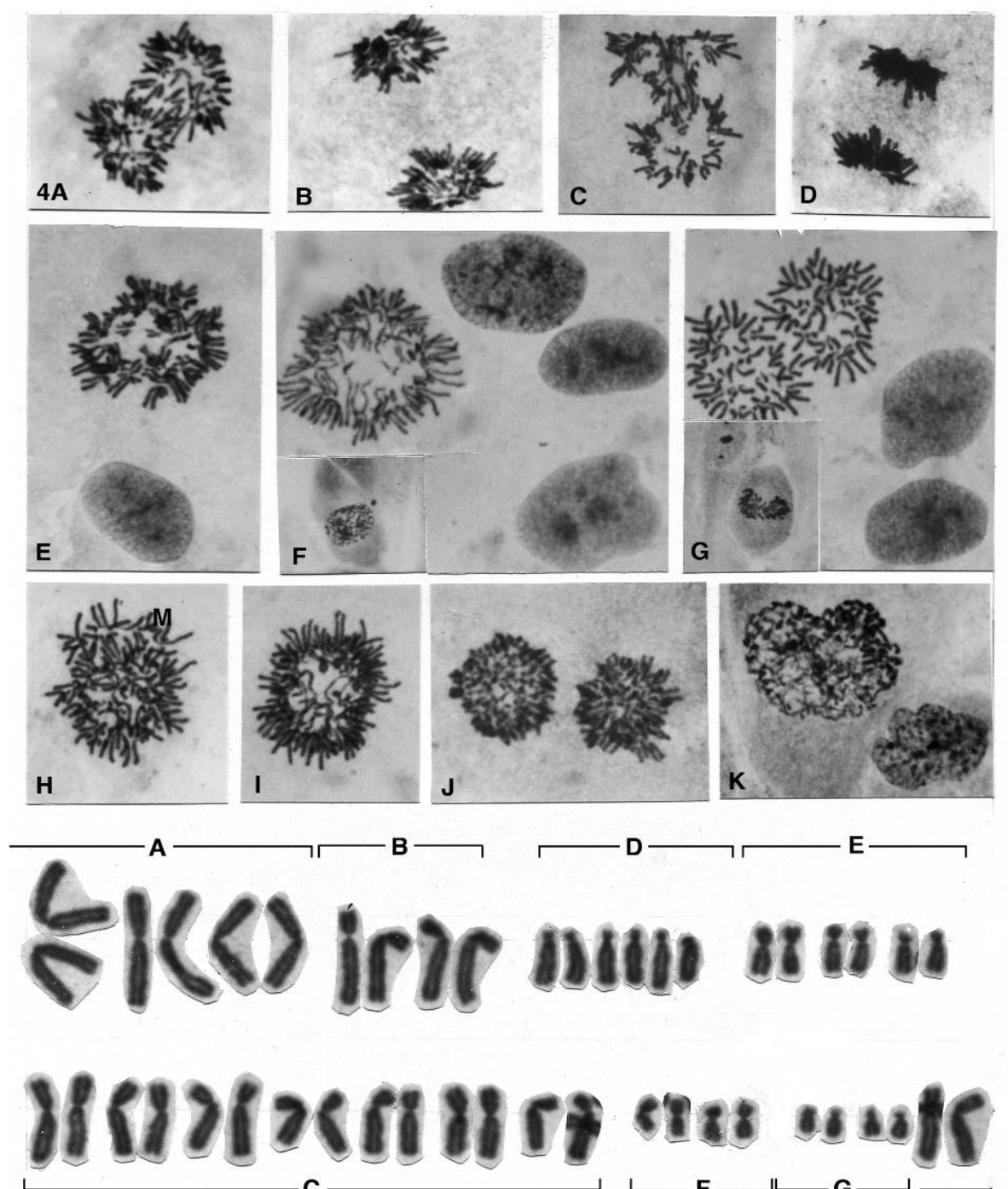

$E$

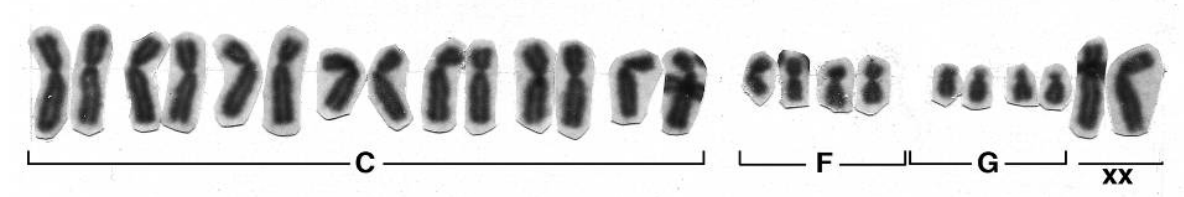

L

Figure 4. Various chromosomal abnormalities of E-dTMC-star-like mitosis in extended passage growths. $(\mathrm{ABCD})$ normal stars in ana- to telophase and one altered division in telophase, but with retained whole complement segregations (C) compared to normal mitotic telophase (D). (EFG) premature chromatid separations and abnormal anaphase stars (G). (HIJ) polyploid stars. (K) polyploid, whole genomic separations in a prophase cell. Inserts in (FG) show perpendicular orientation of endotetraploid cells relative to the cytoskeleton axis. (FG) uneven roundish contours of resting nuclei (achantosis) compare oval fibroblast (Fig.1M). (L) karyotype from $3^{\text {rd }}$ passage growth. All illustrations $2475 \mathrm{x}$

\section{Discussion}

The original experimental goal of an establishment of a method for induction of young cell-response with endopolyploidization for expanded growth-assessments of E-dTMCs was achieved by AAD-treatments. The method was technically simple, repeat-dependable and has probability of happening in people on poor diets. It is 
a one and only known in vitro method for normal cells, which allows molecular characterization of two transient cell division mechanisms leading to two types (E-TMCs and F-dPCs) of aberrantly, proliferating progeny cells. Both types of cells showed hyperplasia-like morphologically proliferative outcomes. The halving of rosette metaphase figures to haploid complements (Figs. 1B-D,G,HL) is supported by chromosomal painting, which showed homologous complements in a diametrically opposite placement in rosette figures (Nagele et al., 1995; Bolzer et al., 2005). All in all, these occurrences add new conceptual considerations of cellular events in cancer-initiation. Both cell-types full-filled the criteria of being derived from normal diploid cells, and also satisfied the fundamental, requirement, in tumorigenesis namely: gain of proliferative advantage (GPA) over the diploid cells of origin (by cell counts and by outgrowths) (Hanahan \& Weinberg, 2011; Coschi \& Dick, 2012). Another fact for GPA is attained cell roundedness linked to perpendicular divisions and to loss of cell contact growth inhibition (see below). Noteworthy, the present GPA was not associated with activation of telomerase which is a late event in tumorigenesis (Feldser et al., 2003), (but being a primary event in immortalization). Interpretively, F-dPCs are homozygous cells from diploid conversion of haploid segregated chromosomal groups. Glass (1957) did karyology of the grouped rat chromosomes $(2 \mathrm{n}=40)$ and showed conclusively haploid genomes, which however, did not succeed for the present human $(2 n=46)$ groupings. Direct proof for human-cell haploidization must await molecular (SNP array) studies of F-dPCs in laboratories with equipment and expertise. (Research in the present-day climate of escalating health-cost ought to be experimental "affordable", - Peters et al., 2012). However, indirectly from existing literature (discussed below) there are several situations in cancers that show haploid conversion to diploidy and also, proliferation of near-haploid cells.

The in vitro hyperproliferation was associated with: change to "streaming" growth patterns (Rosen, 1993; Belge et al., 1998), nuclear and cell roundness-changes (Kazanowska et al., 2004) changed mitotic forms, and focal 3-D growths with cell polarity change, which in vivo is often referred to as nodules (Royer \& Lu, 2011). Previous observations of 3-D tumor-like spheres in old senescent cell cultures (Walen, 2011; 2013), likely had an E-dTMC-origin from the observation of endo-polyploid reductive divisions associated with escape, of polyploid cells from senescent, large flat cells (Walen, 2008). Shabad (1973) summarized microscopic, morphological criteria of pre-cancerous lesions, pointing out that change to nuclear roundness was growth-linked (Sandberg, 1991; Burbano et al., 2000; Kazanowska et al., 2004), and that hyperplasia was a "first stage" followed by change to "focal proliferation". Recently, nuclear morphometry studies showed that nuclear rounded shape-change had predictive value for appearance of abnormal cell proliferation in the oral mucosa (Ahmed et al., 2011), and also in predicting metastasis (Khan et al., 2003).

E-dTMCs exhibited gradual gain in GPA, whereas F-dPCs showed GPA at birth. Suspected homozygosity of these latter cells has support from SNP haplotyping karyology of complete hydatidiform moles (Sarto et al., 1984; Kukita et al., 2013). These studies not only showed homozygosity, but also that haploidy can convert to diploidy. The moles originate from fertilization of an empty egg cell by a haploid X-bearing sperm $(1 \mathrm{n} / 1 \mathrm{C})$, which led to duplication of the haploid genome into homozygous diploid cells that grew into teratomas. And, as for the present study, the diploid teratoma cells divided with changed, star-like mitotic-forms to rounded interphase nuclei (Sarto et al., 1984). Interestingly, 50\% of these benign teratomas changed to chorio-carcinomas, which might be a case for changes to "new" functional genes from genomic-duplications (Wolfe, 2001). Another example of in vivo haploid-associated proliferation is from leukemia and some solid tumors (Drouin et al., 1993; Mitelman, 1988). From Mitelman's Catalog (1988) some karyotype-examples for different cases showing near-haploidy are listed in Table 2. Near to almost complete loss of whole genomes expressing extensive hemizygosity (ALL: 20 monosomic chromosomes) for loss of heterozygosity (LOH) is a remarkable condition for clonal proliferations with GPA. Heim and Mitelman (1998) commented about these occurrences as follows: "How the massive chromosome loss" --- occurs "--- is unknown, and so is the mechanism whereby near-haploidy occurs. --- Many cases have an additional cell line characterized by twice the number of chromosomes in the near haploid clone. This hyperdiploid line presumably arises by secondary endoreduplication", which would lead to noteworthy, significant paradiploid homozygosity from many different whole chromosomal UPDs, also present in various myeloid diseases (Huang et al., 2004; Fitzgibbon et al., 2005; Gondek et al., 2008). The present lack of haploid cell-proliferation (2 haploid metaphase cells observed, but dismissed as incomplete cells) and its occurrence for leukemic near-haploid cells, suggest an environmental difference for proliferative ability between in vitro and in vivo haploid cells. Secreted growth factors from neighboring different cell-types can occur in vivo and not in vitro with only one cell type and synthetic growth-media (Parrinello et al, 2004). Regardless, these in vivo near-haploid genomes are best explained from haploidization of diploid cells (Figs. 1B-H,J-L) (Huskins, 1948; Glass, 1957; Glazko, 2004), because incremental loss of different homologous chromosomes is less likely. 
Table 2. Karyotypes of cells in different cancers

\begin{tabular}{ll}
\hline Leukemia; ALL: & $26, X X,-1,-2,-3,-4,-5,-6,-7,-8,-9,-10,-11,-12,-13,-15,-16,-17,-18,-19,-20,-22$ \\
ALL: & $28, X X,-1,-2,-3,-4,-5,-6,-7,-8,-9,-11,-12,-13,-14,-15,16,-17,-19,-20,-22$ \\
ALL: & $32, X Y,-2,-3,-4,-5,-7,-8,-11,-12,-13,-14,-15,-16,-17,-20$ \\
CML: & $26, X X, t(9: 22),-1,-2,-3,-4,-5,-6,-7,-9,-10,-11,-12,-13,-14,-15,-16,-17,-18,-19,-20$ \\
CML: & $27, X,-Y, t(9: 22),-1,-2,-3,-4,-5,-6,-7,-9,-11,-13,-14,-15,-16,-17,-18,-19,-20,-22$ \\
Kidney: & $31,-X,-X,-1,-2,-3 .-4,-5,-6,-7,-8,-9,-11,-12,-14,-16,-19,-19,-22, t(1: 2)+\mathrm{M}$ \\
Breast: & $35, X X,-1,-3,-4,-5,-7,-8,-10,-11,-12,-13,-14,-15,-16,-17,-18,-19,-20,-21,-22,+8 M s$ \\
Teratoma: Testis: & $67, X Y,+1,+3,+4,+5,+6,+7,+7,+8,+10,+11,+13,+13,+14,+16,+17,+18,+20,+20,+21,+21$ \\
Leukemia; ANLL: & $66, X Y,+1,+1,+2,+4,+6,+6,+7,+8,+8,+10,+11,+11,+12,+12,+18,+19,+21,+21,+22,+22$ \\
\hline
\end{tabular}

Mitleman F. 1988 Catalog of chromosome aberrations in cancer ( $3^{\text {rd }}$ ed.). New York, NY: Alan R. Liss, Inc.

Also in table 2, triploid cells with abnormal proliferation show that occurrence of endo-derived diplochromosomes preceded the karyotypic listed pairs of homologous chromosomes (e.g., $+1,+1)$ which was also a feature in near-diploid breast hyperplasias (Burbano et al., 2000). The 4-chromatid diplochromosomes fall apart to normal-appearing bichromatid chromosomes by chromosomal spreading techniques (Walen, 2007b). Diplochromosomes were observed in untreated leukemia (Houston et al., 1964), and would be observed again, if chromosome spreading techniques were not used in "control" karyology. However, neither of the two mechanisms of genome reductive divisions to genomic changed progeny cells is considered with roles in tumorigenesis.

A new feature in the present study was 3-D growths covered by a substance (Figs 2F, 4E,F), which prevented proper staining, detailing cell shape changes from cell-polarity changes. In tumor-biology cell-shape change, linked to the cytoskeleton system (the perpendicular-division, see Introduction) is claimed to be "important in regulating proliferation" (Yamada, 2003). Tumor cells, besides encased in the extracellular matrix and fibrils "--had an abnormally round shape" with cytoskeleton associated actin filaments distributed on the outside of the cells, which hindered tumor-cell wanderings. Such cover was completely absent over 3-D growth from old senescent cells, which lack cell polarity orientations, and express pleomorphic morphology (Walen 2011; 2013). In vivo senescence-derived relapsed tumor cells after chemo-treatment (with induced polyploidization and reduction), commonly is associated with increased proliferative-robustness and genetic changes, including resistance to original treatment (Puig et al., 2008; Wheatly, 2008 Mosieniak and Sikora, 2010). Are such happenings examples of a senescent "driven" tumorigenesis because of no actin-protein cover over senescent cells? If so, induction of senescence in tumor-therapy would be contra-indicated (Schmitt, 2007; Collado and Serrano, 2010).

The present normal karyotype (Fig. 4L) of E-dTMCs in extended passage-growths, likely involved UPD-formations following loss of laggards (see above) with resultant homozygous-caused LOH for attainment of gradual GPA. This interpretation may well apply to the aneuploid paradox: Cancers arise from normal cells, but normal cells afflicted by aneuploidy show reduced proliferative ability, whereas for cancer development, aneuploidy is associated with gain of proliferative advantage (Gondek et al., 2008; Pfau and Amon, 2012; Holland and Cleveland, 2012). The many cellular changes (Figs. 4E-K) especially, premature chromatid separation can cause asymmetric chromosomal distributions in E-dTMC-growth with probability of aneuploidization-associated LOH. [Both 4-chromatid and 2-chromatid chromosomes can undergo exchanges (Figs. 1E,F). Since such exchanges are between homozygous chromatids, there is heightened probability of unequal exchanges with genetic consequences, as for instance, cryptic mutations and segmental UPDs.]

For E-dTMCs with origin from normal human cells, the essence for this report is the gradual development of GPA and its association with change to focal 3-D growth. Such a sequence of events is postulated by the mutation theory to be due to incremental, accumulated mutations, likely involving loss/change of tumor-inhibiting genes and activation of oncogene (i.e., progression) (Vogelstein and Kinzler, 1993). Today this theory is widely accepted with no more-likely, contender ideas, but it stands to reason that the complex machinery for maintenance of normal proliferative ability can be multi-sensitive to different perturbations for growth behavioral changes (Iversen, 1992; Ganem and Pelman, 2012, Conchi and Dick, 2012). Endopolyploidy, as a response to genomic damage, with potential for aneuploidy and CIN-positive progeny cells, is thus, a highly 
probable mechanism for initiation of tumorigenesis.

This suggestion has support from at least three clear cancer-associated endopolyploidizations: 1) acites tumor cells showed repeated occurrences of 4-chromatid chromosomes in endo-polyploid-divisions (Levan and Hauschka, 1953), 2) genomic reduction of "endo-cells" (4n/8n) back to proliferating diploid cells were associated with increased resistance to the original drug treatment (Puig et al., 2008), and 3) ovarian cancers with giant-endopolyploid cells, depolyploidized by amitotic segregations into multinucleate cells (MNCs), which produced genome reduced, proliferate cancer cells by nuclear budding or MNC-bursting (Zhang et al., 2013). These latter cells were highly tumorigenic in nude mice, demonstrating endopolyploid-associated genomic changes as in 2), and as in 1) these near-diploid cells could renew and repeat the endopolyploid irregular divisions. These are powerful in vivo data that not only suggest, but demonstrate cancer-relevant endopolyploid cycles as a source of genetic variability and, "deregulated proliferation" (Coschi and Dick, 2012). The essence in this latter study is inheritance-caused (Walen, 2012) perpetual, endo-cycles with probability of asymmetric chromosomal distribution, as suggested a century ago (Boveri, 1914; Bignold et al., 2007).

Lastly, the inducer system (AAD) is troublesome, because it has people-possibility of occurrence from poor diets Nutritional inadequacy is especially, for the elderly a possibility, and they have the highest rate of cancer. The finding that deficiency of one single amino acid led to the present dramatic cell changes with similarities to early tumorigenic cytopathic changes is a significant discovery. But not only AA-deficiency needs to be addressed, the larger question is adequate amounts in diets when considering that about $10 \times 10^{10}$ cells (replacement cells) at any time requires AAs for normal mitosis (Conchi and Dick, 2012). Glutamine deficiency, as chosen for the present experiments is especially important for normal metabolism (Deberardinis and Cheng, 2011). Malfunctioning of this machinery can lead to improper cycling of cellular-waste (e.g., kinase m-Tor), which is increasingly being mentioned in a cancer-connection (Tee \& Blenis, 2005). Genome damaged cells left to their own devises, translates into genome damage-associated endopolyploidization with risk of GPA and 3-D cell-growth (Fig. 3D,E). Such cytotoxic lesions can occur from various AA short-comings (Freed and Schatz, 1969), at any time and in any organ containing, normal replacement-growth, but can to a large extent be avoided by change in dietary composition.

\section{Conclusion}

The present study is part of a continued project for the illumination of a possible role of endopolyploid reductive divisions in tumorigenesis. The present goal of securing a young-cell endopolyploid inducing method for observations of long-term proliferative behavior of endo-derived progeny cells was achieved by simple, amino acid deprivation (AAD). Unexpectedly, a second genome reductive mechanism that of haploidization, occurred in recovery growths. Thus, two transient cell division mechanisms led to genome aberrant progenies, expressing the fundamental tumorigenic-associated trait of gain of proliferative advantage (GPA). This was evident from outgrowths of the original normal fibroblast cells with changes to hyperplasia-like growth-morphologies, and 3-D growth with cell polarity change, which are all happenings in early tumorigenesis. Furthermore, cycling reductive endopolyploidy was reported for ovarian cancers, and near-haploid clonal proliferation is known for leukemic cells. In view of these in vitro and in vivo events, a rethinking of future experimental approaches is warranted for informational gain relevant to "beginning" cellular changes in tumorigenesis. Presently, such inquiries most often involve induction of mutations in key-genes associated with cell cycling activity, with the aim of showing their cause of tumor development in mice. The short-coming of this approach is the common use of cancer derived cell lines, which already have genomic changes for proliferative advantage. The present AAD-method for the first time in experimental tumorigenesis (immortalization experimentation excluded, see Discussion) demonstrated that normal, diploid human cells can be the starting material to hyperplasia-like growth (This happening does not speak well for diets poor in AAs). As a model-system it allows molecular identification of genomic changes for GPA, which was hypothesized to derive from homozygous-caused LOH. The future goal for tumorigenic knowledge ought to be reduction in cancer incidence and in cost of therapy, and the present results promises to be a start in such experimental directions.

\section{Acknowledgement}

Cell culturing was part of a quality control project for which I am very grateful to Dr. David Schnurr of the California Department of Health Services.

\section{References}

Agrawal, N., Jiano, Y., Bettegowda, C., Hutfless, S. M., Wang, Y., David, S., ...Meltzer, S. J. (2012). Comparative genomic analysis of esophageal adenocarcinoma and squamous cell carcinoma. Cancer Discovery, 2, 899-905. http://dx.doi.org/10.1158/2159-8290.CD-12-0189 
Ahmed, H. G., Diab, W. M. I., Abdulgafar, S. A., \& Al-Hazimi, A. M. (2011). Role of quantitative nuclear cytomorphometric and NOR dots count in prediction of carcinogenic induced oral cellular proliferative activity. Oncocytology, 1, 1-6. http://dx.doi.org/10.5348/ocyj-2011-1-OR-1

Bakhoum, S. F., Genovese, G., \& Compton, D. A. (2009). Deviant kinetochore microtubule dynamics underlie chromosomal instability. Curr Biol, 19, 1937-1942. http://dx.doi.org/10.1016/j.cub.2009.09.055

Bakhoum, S. F., \& Compton, D. A. (2012). Chromosomal instability and cancer: a complex relationship with therapeutic potential. $J$ Clin Invest, 122, 1138-1143. http://dx.doi.org/10.1172/JCI59954

Barrett, M. T., Pritchard, D., Palanca-Wessels, C., Anderson, J., Reid, B. J., \& Rabinovitch, P. S. (2003). Molecular phenotype of spontaneously arising 4N (G2-tetraploid) intermediates of neoplastic progression in Barrett's esophagus. Cancer Res, 63, 4211-4217.

Belge, G., Roque, L., Soares, J., Bruckmann, S., Thode, B., Fonseca, E., ... Bullerdiek, J. (1998). Cytogenetic investigations of 340 thyroid hyperplasias and adenoma revealing correlations between cytogenetic findings and histology. Cancer Genet Cytogenet. 101, 42-48. http://dx.doi.org/10.1016/S0165-4608(97)00057-5

Bignold, L. P., Coghlan, B. L. D., \& Jersmann, H. P. A. (2007). Context, comments and translations, P 315. In David von Hansemann (Ed.), Contributions to oncology. Birkhauser Verlag, Basel, Switzerland.

Blagosklonny, M. V. (2003). Cell immortality and hallmarks of cancer. Cell Cycle, 2, 296-299. http://dx.doi.org/10.4161/cc.2.4.470

Bolzer, A., Kreth, G., Solovei, I., Koehler, D., Saracoglu, K., Fauth, C., ... Cremer, T. (2005). Three-dimensional maps of all chromosomes in human male fibroblast nuclei and prometaphase rosettes. PLoS Biol, 3, 0826-0842.

Boveri, T. (1914). Zur frage der Entstehung maligner Tumoren. Jena: Fischer

Burbano, R. R., Medeiros, A., Medeiros de Amorim, M. I., Lima, E. M., Mello, A., Neto, J. B., \& Casartelli, C. (2000). Cytogenetics of epiyhelil hyperplasias of the human breast. Cancer Genet Cytogenet, 119, 62-66. http://dx.doi.org/10.1016/S0165-4608(99)00175-2

Collado, M., \& Serrano, M. (2010). Senescence in tumors: evidence from mice and humans. Nat Rev Cancer, 10, 51-57. http://dx.doi.org/10.1038/nrc2772

Coschi, C. H., \& Dick, F. A. (2012). Chromosome instability and deregulated proliferation: an unavoidable duo. Cell Mol Life Sci, 69, 2009-2024. http://dx.doi.org/10.1007/s00018-011-0910-4

Davoli, T., Denchi, E. L., \& de Lange, T. (2010). Persistent telomere damage induces bypass of mitosis and tetraploidy. Cell, 141, 81-93. http://dx.doi.org/10.1016/j.cell.2010.01.031

Deberardinis, R. J., \& Cheng, T. (2010). Q's next: the diverse functions of glutamine in metabolism, cell biology and cancer. Oncogene, 29, 313-324. http://dx.doi.org/10.1038/onc.2009.358

Drouin, V., Viguie, F., \& Debesse, B. (1993). Near-haploid karyotype in a squamous cell lung carcinoma. Genes Chrom Cancer, 7, 209-212. http://dx.doi.org/10.1002/gcc.2870070405

Erenpreisa, J., Salmina, K., Huna, A., Kosmacek, E. A., Cragg, M. S., Ianzini, F., \& Anisimov, A. (2011). Polyploid tumor cells elicit paradiploid progeny through depolyploidizing divisions and regulated autophagic degradation. Cell Biol Int, 35, 687-695. http://dx.doi.org/10.1042/CBI20100762

Fan, H. H., Wang, J., Potanina, A., \& Quake, S. R. (2011). Whole-genome molecular haplotyping of single cells. Nature biotech, 29, 51-57. http://dx.doi.org/10.1038/nbt.1739

Feldser, D. M., Hackett, J. A., \& Greider, C. W. (2003). Telomere dysfunction and initiation of genome instability. Nat Rev Cancer, 3, 623-627. http://dx.doi.org/10.1038/nrc1142

Fitzgibbon, J., Smith, L.-L., Raghavan, M., Smith, M. L., Debernardi, S., Skoulakis, S., ... Young, B. D. (2005). Association between uniparental disomy and homozygous gene mutation in acute myeloid leukemias. Cancer Res, 65, 9252-9154. http://dx.doi.org/10.1158/0008-5472.CAN-05-2017

Freed, J. J., \& Schatz, S. A. (1969). Cromosome aberrations in cultured cells deprived of single essential amino acid. Exp Cell Res, 55, 393-409. http://dx.doi.org/10.1016/0014-4827(69)90574-6

Galipeau, P. C., Cowan, D. S., Sanchez, C. A., Barrett, M. T., Emond, M. J., Levine, Ds., ... Reid, B. J. (1996). $17 \mathrm{p}$ (p53) allelic loss, $4 \mathrm{~N}$ (G2/tetraploid populations) and progression to aneuploidy in Barrett's esophagus. Proc Natl Acad Sci USA, 93, 7081-7084 
Ganem, N. J., \& Pellman, D. (2012). Linking abnormal mitosis to the acquisition of DNA damage. J. Cell Biol, 199, 871-881. http://dx.doi.org/10.1083/jcb.201210040

Geigl, J. B., Obenauf, A. C., Schwarzbraun, T., Speicher, M. R. (2008). Defining “chromosomal instability”. Trends in Genetics, 24, 64-69. http://dx.doi.org/10.1016/j.tig.2007.11.006

Glass, E. (1957). Das Problem der Gesonderung in den Mitosen unbehandelter Rattenlebern. Chromosoma, 8, 468-492. http://dx.doi.org/10.1007/BF01259515

Glazko, T. T. (2004). Chromosomes subdividing to haploid sets in diploid metaphase plated of some mammalian species. In: Proc. $15^{\text {th }}$ Int Chromosome Conf. p63.

Gondek, L. P., Tiu, R., O’Keefe, C. L., Sekeres, M. A., Theil, K. S., \& Maciejewski, J. P. (2008). Chromosomal lesions and uniparental disomy detected by SNP arrays in MDS, MDS/MPD, and MDS-derived AML. Blood, 111, 1534-1542. http://dx.doi.org/10.1182/blood-2007-05-092304

Goodyear, S. M., Amatangelo, M. D., \& Stearns, M. E. (2009). Role of tumor initiating cells in prostate cancer. Trends in Cancer Res, 5, 71-88.

Hanahan, D., \& Weinberg, R. A. (2011). Hallmarks of cancer: the next generation. Cell, 144, 646-674. http://dx.doi.org/10.1016/j.cell.2011.02.013

Hardy, P. A., \& Zacharias, H. (2005). Reappraisal of the Hanseman-Boveri hypothesis on the origin of tumors. Cell Biol Int, 29, 983-992. http://dx.doi.org/10.1016/j.cellbi.2005.10.001

Hayflick, L., \& Moorhead, P. S. (1961). The serial cultivation of human diploid cell strains. Exp Cell Res, 25, 585-621. http://dx.doi.org/10.1016/0014-4827(61)90192-6

Heim, S., \& Mitelman, F. (1995). Cancer Cytogenetics: Chromosomal and molecular genetic aberrations of tumor cells ( $2^{\text {nd }}$ Ed.). New York, NY: Wiley-Liss, Inc.

Holland, A. J., \& Cleveland, D. W. (2012). Losing balance: the origin and impact of aneuploidy in cancer. EMBO, 13, 501-514. http://dx.doi.org/10.1038/embor.2012.55

Houston, E. M., Levin, W. C., \& Ritaman, S. E. (1964). Endoreduplication in untreated early leukemia. Lancet, ii, 496-497. http://dx.doi.org/10.1016/S0140-6736(64)90451-9

Huang, J., Wei, W., Zhang, J., Liu, G., Bignell, G. R., Stratton, M. R., ... Shapero, M. H. (2004). Whole genome DNA copy number changes identified by high density oligonuleotide arrays. Human Genet, 1, $287-299$.

Hunding, A. (1981). Possible prepatterns governing mitosis: the mechnism of spindle-free chromosome movement in Aulacantha scolymantha. J Ther Biol, 89, 353-385. http://dx.doi.org/10.1016/0022-5193(81)90357-X

Huskins, C. L. (1948). Segregation and reduction in somatic tissues. I. Initial observations on Allium cepa. $J$ Hered, 39, 310-325.

Iversen, O. H. (1992). Is high rate of cell proliferation carcinogenic in itself? Human Exp Toxicol, 11, 437-441. http://dx.doi.org/10.1177/096032719201100601

Kazanowska, B., Jelen, M., Reich, A., Tarnawski, W., \& Chybicka, A. (2004). The role of nuclear morphometry in prediction of prognosis for rhabdomyosarcoma in children. Histopathology, 45, 325-359. http://dx.doi.org/10.1111/j.1365-2559.2004.01948.x

Khan, M. A., Walch, P. C., Miller, M. C., Bales, W. D., Epstein, J. I., Mangold, L. A., ... Veltri, R. W. (2003). Quantitative alterations in nuclear structure predict prostate carcinoma distant metastasis and death in men with biochemical recurrences after radical prostatectomy. Cancer, 98, 258-2591. http://dx.doi.org/10.1002/cncr.11852

Kukita, Y., Miyatake, K., Stokowski, R., Hinds, D., Higasa, K., Wake, N., ... Hayashi, K. (2013). Genome-wide definitive haplotypes determined using a collection of complete hydatidiform moles. Genome Res, 15, 1511-1518. http://dx.doi.org/10.1101/gr.4371105

Lee, H. O., Davidson, J. M., \& Duronio, R. J. (2009). Endoreplication: polyploidy with a purpose. Genes Dev, 23, 2461-2477. http://dx.doi.org/10.1101/gad.1829209

Lengauer, C., Kinzler, K. W., \& Vogelstein, B. (1998). Genetic instabilities in human cancers. Nature, 396, 623-646. http://dx.doi.org/10.1038/25292

Levan, A., \& Hauschka, T. S. (1953). Endomitotic reduplication mechanisms in ascites tumors of the mouse. $J$ 
Nat Cancer Inst, 14, 1-43.

Lipkin, M. (1988). Biomarkers of increased susceptibility to gastrointestinal cancer: New application to studies of cancer prevention in human subject. Cancer Res, 48, 235-245.

Manchado, E., Guillamot, M., \& Malumbres, M. (2012). Killing cells by targeting mitosis. Cell Death Differen, 19, 369-377. http://dx.doi.org/10.1038/cdd.2011.197

Mitelman F (1988) Catalog of chromosome aberrations in cancer. Alan Liss, Inc., New York, NY

Mosieniak G, Sikora E. Polyploidy: The link between senescence and cancer. Curr Pharmaceut Design 2010; 16:734-74. http://dx.doi.org/10.2174/138161210790883714

Nagele, R., Freeman, T., McMorrow, L., \& Lee, H. Y. (1995). Precise spatial positioning of chromosomes during prometaphase: Evidence for chromosomal order. Science, 270, 1831-1835. http://dx.doi.org/10.1126/science.270.5243.1831

Parrinello, S., Coppe, J. P., Krtolica, A., \& Campisi, J. (2004). Stromal-epithelial interactions in aging and cancer: senescent fibroblasts alter epithelial cell differentiation. J Cell Science, 118, 485-496. http://dx.doi.org/10.1242/jcs.01635

Peters, B. A., Kermani, B. G., Sparks, A. B., Alferov, O., Alexeev, A., Jiang, Y., ... Drmanac, R. (2012). Accurate whole-genomic sequencing and haplotyping from 10 to 20 human cells. http://dx.doi.org/10.1038/nature11236

Pfau, S. J., \& Amon, A. (2012). Chromosomal instability and aneuploidy in cancer: from yeast to man. $E M B O$ reports, 13, 515-527. http://dx.doi.org/10.1038/embor.2012.65

Phillip, R., Campbell, E., \& Wheatley, D. N. (2003). Arginine deprivation, growth inhibition and tumor cell death: 2. Enzymatic degradation of arginine in normal and malignant cell cultures. Brit $J$ Cancer, 88 , 613-623. http://dx.doi.org/10.1038/sj.bjc.6600681

Puig, P. E., Guilly, M. N., Bouchot, A., Droin, N., Cathelin, D., Bouyer, F., ... Chauffert, B. (2008). Tumor cells can escape DNA-damaging cisplatin through DNA endoreduplication and reversible polyploidy. Cell Biol Int, 32, 1031-1043. http://dx.doi.org/10.1016/j.cellbi.2008.04.021

Raikov, I. B. (1982). The protozoan nucleus, morphology and evolution. In: Alfert M, Beermann W, Goldstein L, Porter KR, Sitte P (Eds.). Cell Biology Monographs. Vol. 9. Wien-New York: Springer-Verlag: pp 209-214

Rosen, P. P. (1993). Proliferative breast "disease": An unresolved diagnostic dilemma. Cancer, 71, 3798-3807. http://dx.doi.org/10.1002/1097-0142(19930615)71:12<3798::AID-CNCR2820711203>3.0.CO;2-S

Royer, C., \& Lu, X. (2011). Epithelial cell polarity: a major gatekeeper against cancer? Cell Death Differen, 18, 1470-1477. http://dx.doi.org/10.1038/cdd.2011.60

Sandberg, A. A. (1991). Chromosome abnormalities in human cancer and leukaemia. Mutation Res, 247, 231-240. http://dx.doi.org/10.1016/0027-5107(91)90019-K

Sarto, G. E., Stubblefield, P. A., Lurain, J., \& Therman, E. (1984). Mechanisms of growth in hydatidiform moles. Am J Gynecol, 148, 1014-1023.

Saunders, W. S., Shuster, M., Huang, X., Gharaibe, B., Enyenihi, A. H., Petersen, I., \& Gollin, S. M. (2000). Chromosomal instability and cytoskeletal defects in oral cancer. Proc Natl Acad Sci USA 97:303-308. http://dx.doi.org/10.1073/pnas.97.1.303

Schmitt, C. A. (2007). Cellular senescence and cancer treatment. Biochim Biophys, 1775, 5-20.

Shabad, L. M. (1973). Precancerous morphological lesions. JNCI, 50, 1421-1428.

Sheltzer, J. M., \& Amon, A. (2011). The aneuploidy paradox: cost and benefits of an incorrect karyotype. Trends in Genetics, 27, 446-453. http://dx.doi.org/10.1016/j.tig.2011.07.003

Swanson, C. P. (1957). Cytology and Cytogenetics. Prentice-Hall, Inc., Englewood Cliffs, N.J. USA

Tee, A. R., \& Blenis, J. (2005). mTOR, translational control and human disease. Semin Cell Dev Biol, $16,29-37$. http://dx.doi.org/10.1016/j.semcdb.2004.11.005

Vogelstein, B., \& Kinzler, K. W. (1993). The multistep nature of cancer. Tig, 9, $138-141$. http://dx.doi.org/10.1016/0168-9525(93)90209-Z

Walen, K. H. (2006). Human diploid fibroblast cells in senescence: Cycling through polyploidy to mitotic cells. In Vitro cell Dev Biol Animal, 42, 216-224. http://dx.doi.org/10.1290/0603019.1 
Walen, K. H. (2007a). Bipolar genome reductional division of human near-senescent, polyploid fibroblast cells. Cancer Genet Cytogenet, 173, 43-50. http://dx.doi.org/10.1016/j.cancergencyto.2006.09.013

Walen, K. H. (2007b). Origin of diplochromosomal polyploidy in near-senescent fibroblast cultures: Heterochromatin, telomeres and chromosomal instability (CIN). Cell Biol Int, 31, 1447-1455. http://dx.doi.org/10.1016/j.cellbi.2007.06.015

Walen, K. H. (2008). Genetic stability of senescence reverted cells: Genome reduction division of polyploid cells, aneuploidy and neoplasia. Cell Cycle, 7, 1623-1629. http://dx.doi.org/10.4161/cc.7.11.5964

Walen, K. H. (2009). Spindle apparatus uncoupling in endo-tetraploid asymmetric division of stem and non-stem cells. Cell Cycle, 8, 3234-3237. http://dx.doi.org/10.4161/cc.8.19.9570

Walen, K. H. (2010). Mitosis is not the only distributor of mutated cells: non-mitotic endopolyploid cells produce reproductive genome reduced cells. Cell Biol Int, 34, 867-872. http://dx.doi.org/10.1042/CBI20090502

Walen, K. H. (2011). Normal human cell conversion to 3-D cancer-like growth: genome damage, endopolyploidy, senescence escape, and cell polarity change/loss. J Cancer Thera, 2, 181-189. http://dx.doi.org/10.4236/jct.2011.22023

Walen, K. H (2012). Genome reversion process of endopolyploidy confers chromosome instability on the descendent diploid cells. Cell Biol Int, 36, 1-9. http://dx.doi.org/10.1042/CBI20110052

Walen, K. H. (2013). Senescence arrest of endopolyploid cells renders senescence into a mechanism for positive tumorigenesis. In: Tumor Dormancy and Cellular Quiescence and Senescence. Vol. 1: Aging, Cancer, and Noncancer Pathologies. http://dx.doi.org/10.1007/978-94-007-5958-9_18

Wheatley, D. N. (2008). Growing evidence of the repopulation of regressed tumors by the division of giant cells. Cell Biol Int, 32, 1029-1030. http://dx.doi.org/10.1016/j.cellbi.2008.06.001

Wolfe, K. H. (2001). Yesterday's polyploids and the mystery of diploidization. Nature Rev Genetics, 2, 333-341. http://dx.doi.org/10.1038/35072009

Yamada, K. M. (2003). Tumor jailbreak. Nat 424:889-890. http://dx.doi.org/10.1038/424889a

Zhang, J., Wang, X., Zhao, Y., Chen, B., Suo, G., \& Dai, J. (2006). Nedoplastic transformation of human diploid fibroblasts after long-term serum starvation. Cancer Letter, 243, 101-108. http://dx.doi.org/10.1016/j.canlet.2005.11.022

Zhang, S., Mercado-Uribe, I., Sun, B., Kuang, J., \& Liu, J. (2013). Generation of cancer-stem-like cells through the formation of polyploid giant cancer cells. Oncogene, 1-13. http://dx.doi.org/10.1038/onc.2013.96

Zybina, E. V., \& Zybina, T. G. (1996). Polytene chromosomes in mammalian cells. Int Rev Cytol, 165, 53-119. http://dx.doi.org/10.1016/S0074-7696(08)62220-2

Zybina, E. V., Zybina, T. G., Bogdanova, M. S., \& Stein, G. I. (2005). Whole-genome chromosome distribution during nuclear fragmentation of giant trophoblast cells ofMicrotus rossiae-meridonalis studies by use of gonosomal chromatin arrangement, Cell Biol Int, 29, 1066-1070. http://dx.doi.org/10.1016/j.cellbi.2005.10.014

Yang, H., Chen, X., \& Wong, W. H. (2011). Completely phased genome sequencing through chromosome sorting. Proc Natl Acad Sci, 108, 12-17. http://dx.doi.org/10.1073/pnas.1016725108

\section{Copyrights}

Copyright for this article is retained by the author(s), with first publication rights granted to the journal.

This is an open-access article distributed under the terms and conditions of the Creative Commons Attribution license (http://creativecommons.org/licenses/by/3.0/). 